possible light on to the vexed question of why and how the ancient supercontinents split apart in the first place. Prior to the opening of the Red Sea, the eastern boundary of the then African-Arabian plate would have had a re-entrant corner to the east of the tip of what is now the Somali Republic. According to Harper such a corner would magnify the (tensile) stresses locally, facilitating the formation of a crack. Certainly a crack developed in this case, propagating towards the southern tip of Arabia and then, with a change of direction, up what was to become the Red Sea (a course presumably dictated partly by local geology and partly by the forces pulling towards the subduction zone beneath the Zagros mountains in the northeast). But this change of direction would have produced a new re-entrant corner at the southern tip of Arabia, a new local concentration of stress and (as evidently occurred) a new split forming the east African rift.

Would it be possible to explain the gradual break up of Gondwanaland (or even Pangaea) entirely in terms of a series of cracks propagating from reentrant boundary corners, perhaps aided in many cases (once the process got going) by pulling at appropriately situated subduction zones?

\section{Faithful transcription in vitro}

\section{from T. Barrett}

RECENTLY much interest has focused on reproducing in vitro the transcriptional ability of native chromatin. The manipulation of chromatin during its isolation, most notably shearing to render it soluble, is liable to alter its template activity, and it is reasonable to be sceptical about the relationship that in vitro transcription products bear to RNA synthesised in vivo. De Pomerai et al. (Eur. J. Biochem., 46, 461; 1974) demonstrated that chromatin prepared by various published methods differed both in structure, as defined by the DNA : RNA : protein ratio, in template activity using both endogenous and added homologous RNA polymerase, and in the size of transcript produced. Shearing of chromatin resulted in a decrease in transcript size and an altered size distribution on formamide sucrose gradients. These authors suggested that extraction of chromatin and RNA polymerase from eukaryotic cells may remove factors necessary for in vivo transcription and subsequent processing of RNA.

More recently Noll et al. (Science, $187,1203 ; 1975)$ demonstrated that the structure of native chromatin is easily destroyed when chromatin is prepared by conventional methods involving shear. Such chromatin does not have the repeating structure shown by native chromatin when digested by micrococcal nuclease. They have developed a technique whereby the native structure is retained, which involves slight nuclease digestion of isolated nuclei followed by lysis in hypotonic buffer. The resulting chromatin shows the same 200 base pair repeating unit found in nuclear chromatin. But they point out that chromatin, even when prepared by this comparatively gentle method, is not necessarily identical with chromatin in the nucleus.

The most direct approach to the study of fidelity of transcription in vitro has been to search for specific RNA sequences in the transcription product. Paul and Gilmour ( $J$. molec. Biol. 34,305 ; 1968) first indicated that organspecificity was retained in isolated chromatin, but their hybridisation techniques only provided information about RNA transcribed from reiterated DNA sequences. The use of reverse transcriptase to copy specific messenger RNAs into highly radioactive complementary DNAs has, however, made it possible for Axel et al. (Proc. natn. Acad. Sci. U.S.A., 70, 2029, 1973) and Gilmour and Paul (Proc. natn. Acad. Sci. U.S.A., 70, $3440 ; 1973$ ) to demonstrate that RNA transcripts of chromatin isolated from erythropoietic tissues contain globin-specific RNA sequences.

These studies were performed using bacterial RNA polymerase and indicated nothing about the accuracy of transcription of the globin genes on isolated chromatin. Steggles et al. (Proc. natn. Acad. Sci. U.S.A., 71, 1219; 1974) compared the transcription of globinspecific RNA sequences on rabbit bone marrow chromatin using both bacterial and mammalian form B RNA polymerase. Both enzymes transcribed globin-specific RNA sequences, but with mammalian enzyme globin sequences represented a higher proportion of the RNA synthesised $10.05 \%$ for mammalian as opposed to $0.016 \%$ for the bacterial enzyme). The use of homologous rather than bacterial polymerase, however, does not provide the complete answer to the problem of accurate in vitro transcription. Honjo and Reeder (Biochemistry, 13, 1896; 1974) found that added homologous RNA polymerase transcribed the rRNA genes of Xenopus laevis chromatin in an aberrant manner. The polymerase transcribed both strands and the spacer regions and in fact was no more accurate than bacterial polymerase in this respect.

In a recent paper Marzluff and Huang (Proc. notn. Acad. Sci. U.S.A., $72,1082 ; 1975)$ claim to have achieved faithful transcription of low molecular weight RNA species using the endogenous RNA polymerase of mouse myeloma chromatin. Chromatin was isolated from nuclei by lysis in hypotonic buffer and then sheared by passing it through a narrow gauge syringe needle. The accuracy of transcription of the 5S RNA and tRNA genes in vitro was confirmed by the correct size of the transcripts, $5 \mathrm{~S}$ for the $5 \mathrm{~S}$ gene and $4.5 \mathrm{~S}$ for the precursor of $4 \mathrm{~S}$ tRNA. In addition correct strand transcription was indicated for the 5S RNA gene since the transcript hybridised only to the + strand of Xenopus laevis 5S DNA. Although Zylber and Penman (Proc. natn. Acad. Sci. U.S.A., 68, $2861 ; 1972)$ and Reeder and Roeder $(J$. molec. Biol., 67, 433; 1972) had previously shown that rRNA is faithfully transcribed in isolated nuclei, it is of interest that in isolated chromatin where half the nuclear protein and $90 \%$ of the nuclear RNA is removed, an accurate transcript of the $5 \mathrm{~S}$ and $4 \mathrm{~S}$ genes can still be obtained. There are differences, however, between isolated nuclei and isolated chromatin. Marzluff and Huang found that the chromatin system was much less stable as regards RNA synthetic activity. Activity is rapidly lost at $4{ }^{\circ} \mathrm{C}$ and is completely destroyed by freezing. The average molecular weight of the total RNA transcript from chromatin is lower, probably because of the shearing process employed during chromatin preparation, although this does not appear to affect the low molecular weight RNAs which are still synthesised accurately. In chromatin no 4S RNA was identified, although a $4.5 \mathrm{~S}$ precursor species was synthesised. This contrasts with the nuclei where some 4S RNA is produced and suggests that the enzyme responsible for cleaving the $4.5 \mathrm{~S}$ precursor is missing or inactive in the chromatin preparation.

In spite of the differences, the preferential incorporation of $\gamma_{-}{ }^{32} \mathrm{P}-\mathrm{GTT}$ into $5 \mathrm{~S}$ and $4.5 \mathrm{~S}$ RNA suggests that these species are being reinitiated and transcribed continually in vitro. The genes for 5S and 4S RNA represent only a small portion of the nuclear DNA $(0.001 \%$ and $0.005 \%$ respectively) but they account for nearly 1,000 times that amount of RNA, indicating that strict template restriction is maintained in vitro. Marzluff and Huang suggest that the structure of these genes in chromatin and their interaction with factors other than polymerases may result in their great activity in vitro. This may explain why isolated polymerases, whether homologous or bacterial, do not show the same extensive restriction when used to transcribe chromatin. Such studies emphasise that the native structure of chromatin and its intimate association with some, as yet unknown, labile factors may be absolutely essential for the accurate transcription of genes in vitro. 